\title{
扁平城市：数字通信技术进步背景下世界城市发展的 新趋向及对中国的启示
}

Flat City: Development Trend of World Cities Under the Influence of Digital Communication Technology Progress and Its Enlightenment to China

摘要: 世界范围内不同国家城市化呈现出不同类型。既有经历了第一、 二次城市浪潮而形成的成熟城市, 也有正在发展的新兴城市。数字通信 技术的进步影响了成熟城市与新兴城市的功能和形态，它们的发展暗示 着一种新的城市趋向——扁平城市”。扁平城市是在城市中任何区域能 够获得经济与信息自由的城市, 它避免长距离延伸基础设施, 降低人均 碳排放量。数字通信技术的发展也影响着中国人的生活：城市组织方式由 “职能中心”向“综合组团”转变、道路由“运输型”向“生活型”转变以 及发展彼此关联、功能相互补充的小型城市中心将有助于中国城市的扁平 化。扁平城市对土地与能源相对紧张的中国城市的发展具有重要参考意义。

Abstract: Different countries have different types of urbanization. There are "mature cities" that have grown during the first and second urbanization waves and "emerging cities" that are currently forming. These two types of cities' form and function have been changed because of the development of Digital Communication Technologies and therefore, a new type of city development, one we might call the "flat city", appears. "Flat city" is a city within which economic development and information freedom can be achieved anywhere. It helps to avoid the over extension of infrastructure and reduce per capita GHG. Meanwhile, the Chinese people's lives are influenced by Digital Communication Technologies. Due to the development of flat city, urban structure transforms from once dominated by a "functional center" to one dominated by "comprehensive community clusters"; urban road function changes from "transportation priority" to "life priority"; and more small-scale city centers which are connected each other and complemented in functions are created. The concept of flat city is an important reference for the development of Chinese cities whose land and energy resource are under increasing strain.

关键词: 城市化; 成熟城市; 新兴城市; 扁平; 数字化通信技术

Keywords: Urbanization; Mature City; Emerging City; Flat;

Digital Communication Technology

国家自然科学基金资助项目（51508516）; 国家留学基金委资助项目（留金 法 [2014]5035 号)

作者: 黄晶, 博士, 郑州大学建筑学院, 副教授。jingdraa@163.com 帕特里克·康登 (通信作者), 加拿大英属哥伦比亚大学建筑与风景园 林学院教授。pcondon@sala.ubc.ca
20 世纪 90 年代以来, 中国城市表现出的快速城市化 态势对国家资源与环境形成了巨大与持久的压力。中国人 口众多, 人均资源相对稀少, 城市扩张不仅消耗了大量的 土地与能源, 同时还因基础设施建设量增加、机动车使用 频率提高等原因, 加剧了环境污染。

世界范围内的城市化是一个普遍的现象。2013 年, 全 球越过了一个重要的界限一一超过 $50 \%$ 的人口居住在城 市, 到 2050 年这一数值将超过 $75 \%{ }^{[1]}$ 。

各个国家的城市化呈现出不同类型：有经历了第一 次与第二次工业技术发展浪潮的成熟城市, 也有正在形 成的新兴城市。本文从数字化通信技术 (DCT: Digital Communication Technologies) 如何影响与改变城市形式的 角度, 挖掘成熟城市与新兴城市之间的差异, 提出了一种 扁平城市的城市发展趋向, 并讨论了扁平城市对中国城市 发展的启示。

\section{1 三次城市浪潮与成熟城市}

\section{1 第一次和第二次城市浪潮}

世界上最为古老与知名的城市都曾经历了由机械能 (machine energy) 驱动发展的阶段。在这个过程中, 通信 起到了重要的作用。在纽约、伦敦等大都市的第一次发展 浪潮中, 工厂、有轨电车、家庭以及地铁所使用的能源大 部分来自于燃煤蒸汽。那时, 电报以及日报等大型媒体机 构是通信的主要形式; 到了 20 世纪后半叶, 石油内燃机 带来了第二次浪潮城市。个人的交通方式转化为机动车交 通, 而铁路与卡车主要用于货运。这一时期, 通信的形式 主要是双向的电话通信以及单向的广播电视网络。

在第二次城市浪潮蓬勃发展时期, 工作与居住等主 要城市功能区之间的距离被拉长, 郊区大多是工作者睡觉 的地方。人们长距离的驾驶, 以及郊区独立住宅对能源 
的低效利用, 导致了人均能源消耗与人均温室气体 (GHG: Greenhouse Gas）排放量的提高。

在北美, 大多数工作职位位于中心城区, 这样的城市布 局引发了城市中交通设施的大量建设。二战结束后, 美国迎 来了一个前所未有的高速公路投资时代。仅仅 20 多年, 洛 杉矶地区就变成了一个拥有世界最大高速公路系统的城市, 这个城市是一个完全依赖个人机动交通的世界 ${ }^{[2]}$ (图 1)。

\section{2 第三次城市浪潮与成熟城市 (mature city) 出现}

虽然第三次城市浪潮成型较慢, 近些年却发展快速。20 世纪 80 年代, 伴随着互联网与移动电话的出现, 理论家们 预言一个更为分散与高效的城市将会到来, 这种城市不需要 负担因为城市功能严重分离而产生的区域之间大量交通连接 的成本。但是, 让他们失望的是, 20 世纪八九十年代的远 程交换还仅仅停留在工作者的个人数据交换上, 居住区的蔓 延以及随之而来的通勤距离的增长并没有减弱。

直到智能手机以及家用电脑的普及, 成熟城市慢慢开始 成型。在智能手机的帮助下, 人们几乎可以在任何场所工作 或娱乐。

(1) 驾驶量的减少

多年以来, 美国人均驾驶里程在逐步上升, 美国人每天 在城市及其周边进行越来越长以及越来越大量的通勤活动。 据统计, 2000 年美国人每天平均旅行量是 20 世纪 20 年代 的 10 倍 ${ }^{[3]}$ 。

到了 2005 年, 人们的旅行量开始出现下降的趋势。 2008 年美国引发了全球经济危机, 在之后的几年, 美国经 济一直在持续衰退。到了 2013 年, 美国人的汽车旅行距离 比 2005 年降低了 $9 \%$ 。这个下行趋势在 16 34 岁的年轻人群 中达到了 $23 \%$ 。

智能手机、云计算以及通过固定场所自由获取数据, 促 进了人们出行量的下降。远程办公在持续增长, 2005-2011 年间增长了 $73 \%{ }^{[5]}$ 。即使当工作者坐在相连的办公桌, 他们

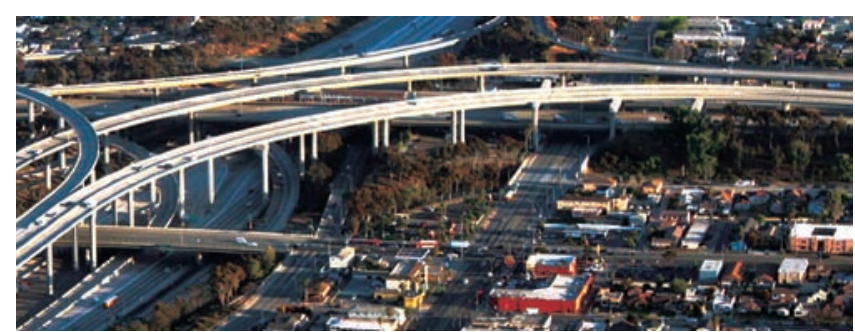

图 1 建于 1961 年的洛杉矶 1-405 公路

资料来源: People for Bikes. Quick builds for better streets: a new project delivery model for U.S. cities[R]. 2016. 3.
也会利用电子方式彼此联系。会议可以采用 “Skype” 或者 其他能够面对面的电子会议技术, 例如思科网真 ${ }^{1}$ 、云视频 (Video Cloud) 等。

在各种数字通信技术 (例如, 在线会议、云计算、电子 邮件以及视频电话）的全力帮助下, 距离不再是问题, 工作 更多地成为一个 “我做的事情, 而不是一个我要去的地点”。 虽然现在大部分依托数字化通信技术的远程工作者每周仍旧 需要去固定的工作场所, 与他们的工作伙伴或者顾客面对面 地交流。但是, 数字化通信技术的发展正在逐渐地减少人们 的通勤旅行 ${ }^{[5]}$, 它用 “可人性” 替代了 “移动性” ${ }^{[6]}$, “远程 工作”与 “在办公室工作”之间正在发生着相互替代与补

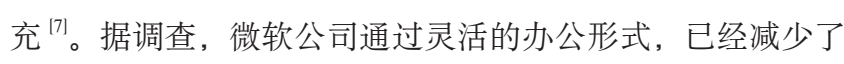
$27 \%$ 的通勤量。永实国际律师事务所每年在差旅上节约了七 位数的花销 ${ }^{[8]}$ 。

现在, 很多人开始习惯于在家工作。这样的现象刺激了 对靠近住所的公共设施的需求, 以及将城郊居住区变成一个 更为完备的可工作、可居住场所的需求。近些年来, 许多大 城市渐渐摆脱了单中心结构, 人们的日常通勤目的地不再仅 仅是往返于老的 CBD, 而是在更为广泛的城市区域中扩散 ${ }^{[9]}$ 。 因此, 单一中心的二次浪潮城市的影响力逐渐式微, 单一文 化的郊区飞地也在加速消减。

(2) 随处可工作

同样的力量将人们从城市中心的办公室推了出来, 也将 人们从家中驱逐了出来。在数据被存储在云端的条件下, 人 们可以自由地在任何时间、任何场所工作一一可能是咖啡馆、 公园的长椅, 以及其他很多可选择的公共场所 (图 2)。

如果将办公室与家比作人们生活的两个极点, 城市越 来越需要处于这两个极点之间的可供选择的工作场所, 在这

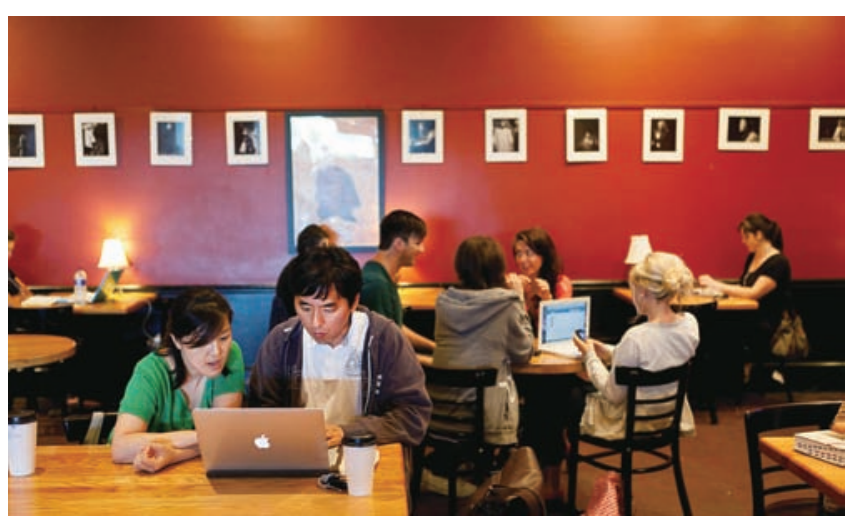

图 2 在咖啡馆工作的人们

资料来源 : Có nên làm việc tại nhà, quán cafe thay vì đến công sở?[EB/OL]. (2015-07-11) [2017-03-15]. http://lienthongchinhquy.com/.

(1) 一种通过结合高清晰度视频、音频和交互式组件, 在网络上创建一种独特的面对面体验的新型技术。 
里人们可以工作, 或者与合作伙伴、客户会面, 这样的场 所被称为 “第三空间”。正如考恩琉斯・麦德维恩 (Cornlius Medvei）所言 “当我们决定去改变我们的工作方式时, “第 三空间, 是一个能够激发更好的合作与思想火花的地方”。 “第三空间” 往往离家不远, 通常是配备 WiFi 的咖啡馆、 酒吧和图书馆, 人们对这类城市空间的需求还在持续增长 ${ }^{[8]}$ 。

这也可以部分地解释, 为什么拥有丰富的步行可达的商 业设施、建造于街车时代的老中心城市以及第一环郊区, 在 美国经济下滑的 2008 年, 它们的价值并没有出现大幅降低 的现象。相对的是, 第三环郊区的平均价值降低了 $30 \%$ 多。

“随处可工作” 促进了更为完整的社区、以及人们全天可以 通过步行、自行车以及短途公共交通往返家庭与服务场所生 活方式的形成。

很明显, 当本地服务与活动在整体经济中所占比例越大 时, 消耗的能源就会越小, 还会导致对交通设施需求的降低 ${ }^{[6]}$, 城市会变得更为可持续。

\section{2 新兴城市}

\section{1 发展中国家城市的发展}

事实上, 在那些新兴城市中发生的类似变化更是令人难 以置信。相较于成熟城市, 拉丁美洲、亚洲以及非洲的人口从 农村到城市的迁徙处于一个更早期阶段, 但是发展很快; 到 2060 年, 将会有 30 亿人口从农村移居到城市 ${ }^{[1]}$ 。在很多城市中, 非法定居点似乎在一夜之间就涌现了出来。快速扩张的定居点 与城市中原有的 “合法” 片区看起来格格不人。

除了正在努力地通过排列式的高层住宅区、蔓延的高速 公路以及 10 车道的城市干道来完成二次城市浪潮的中国之 外, 其他很多新兴城市虽然看起来混乱, 但是它们正在避 免极端的土地使用功能分离, 以及对长距离交通设施依赖 等问题。

在那些以有机形式连续增长的地区, 一种新的更可持续 的城市形式正在涌现, 通信在其中同样起了重要作用。圣保 罗 (São Paulo) 的大部分自建棚户区在十几年前逃避了法律的 管制。对于那些从农村涌向城市的移民，棚户区提供了合理而 安全的环境（图 3)。这里的居民有很高的就业率一一大概有 超过 $50 \%$ 处于工作年龄段的人口被雇佣或者自雇 ${ }^{[10,11]}$ 。小型 创业活动史无前例地活跃。与政府投资的单一功能高层社区相 比较, 这些有机社区具有天生的韧性和适应性。它们能够以一 种前所未有的尺度, 通过自我调整, 促进创业行为的发生。这 样变化的关键何在? 总的来说是数字化通信技术, 尤其是移动 电话。

\section{2 手机带来的一种新自由}

对于很多居民来说, 移动电话的获取可能早于供排水设 施、取暖设施、家具 ${ }^{[12]}$ 。在成熟城市中, 手机是日收人没有 超过 10 美元人的奢侈品, 在新兴城市则不同。在里约热内 卢最大的贫民窟罗西尼亚 (Rocinha) 中, 虽然许多地段缺 乏诸如排水系统等基础服务设施, 但是这里却拥有大量的网 吧, 以及免费的 WiFi 系统 ${ }^{[13]}$ 。

近 10 年, 一个依赖数字化通信技术的全新商业形式出 现了。移动电话促使人们用现代途径从事经济活动。人们不 需要去银行而是通过移动电话就能操作银行业务。经营者与 顾客的关系更为直接——经营者可以按照顾客的要求提供服 务, 顾客因为接受了服务可以多渠道地将钱转给经营者 ${ }^{[1,15]}$ 。 这个过程几乎不需要任何成本来支持基础设施。伴随着这些 城市的发展, 更为成熟的智能电话与互联网接人技术将被更 为广泛地应用 ${ }^{[16]}$ 。

数字化通信技术对这些城市的形态产生了影响。新兴区 域拥有数量众多、功能多样、规模微小的企业——例如大量 自发形成的沿街商业以及一些小型工厂。这些小型的企业往 往与本地需求建立了紧密的联系, 提供的服务多为非正式的。 “它们可以被理解为本地的社会与商业网络” [17]。这样的小 型企业非常的现代, 它们经常集中于街道或集市中, 以便于 顾客和供货商之间建立更为密切的联系, 方便为大量客户提 供更为多样的服务 ${ }^{[18,19]}$ 。数字化通信技术的发展给这些小型 企业创造了更高的自由度和更好的生存条件, 例如经营者与 供货商、消费者之间的远程联系, 为小型企业的多渠道经营 提供了更有效的支持 ${ }^{[20]}$ 。在新兴城市中许多工作机会是这 种小型商业企业创造的, 它们能够为城市低收人者提供低成

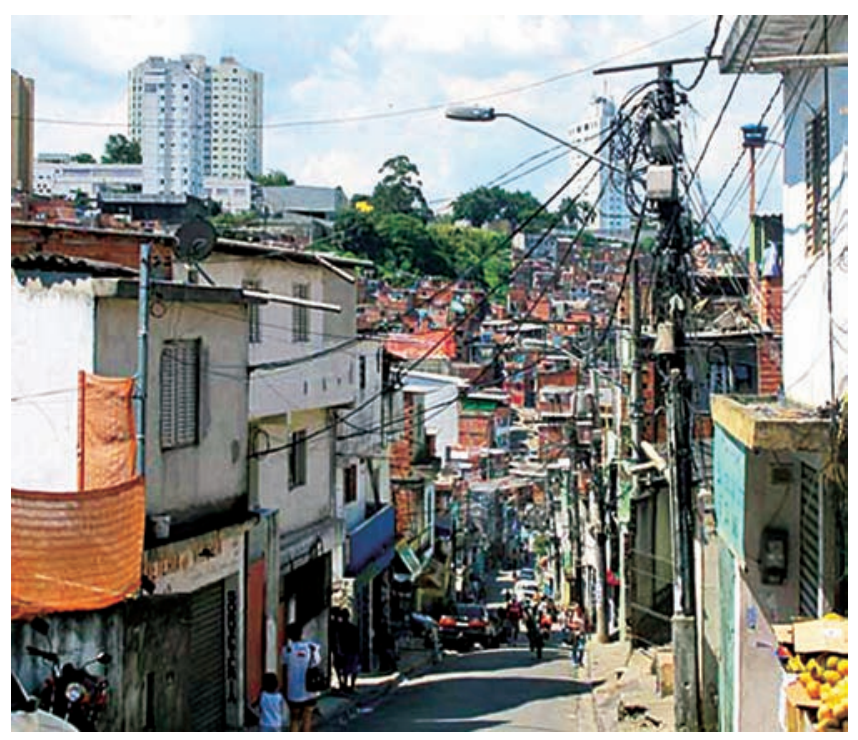

图 3 巴西圣保罗的棚户区

资料来源 : BONADIO L, EM SP. Paraisópolis ainda sofre com problemas de infraestrutura [EB/ OL]. (2011-12-21) [2017-03-15]. http://g1.globo.com/. 
本的服务。经济机会与自由度的增长反映在新兴城市的尺度 中一一狭窄的街道、紧凑的房子以及有机的结构。这样的工 作不仅仅创造了价值, 它们还对人们的生计以及更为广泛的生 存环境产生了影响 ${ }^{[17]}$ 。

2011 年, 扬 - 库里利卡 (Jan Kudlicka) 研究了罗西尼 亚 (Rocinha) 中的 “小农场” (little farm) 片区, 他指出贫 民窟的形成体现出一种根据居住者的需求来建造城市的新方 法。巴西的平民窟从某种意义上讲是现代城市中的乌托邦。 它是一个运行非常良好的自满足系统, 人们彼此熟悉, 互动 频繁。这里没有主要城市中心, 没有集中的工业区以及大型 的购物中心。不同的小型中心彼此连接、功能互补。这样的 结构既有利于人们在步行距离解决生活需求, 又具有较强的 社会稳定性 ${ }^{11}{ }^{[21]}$ (图 4)。
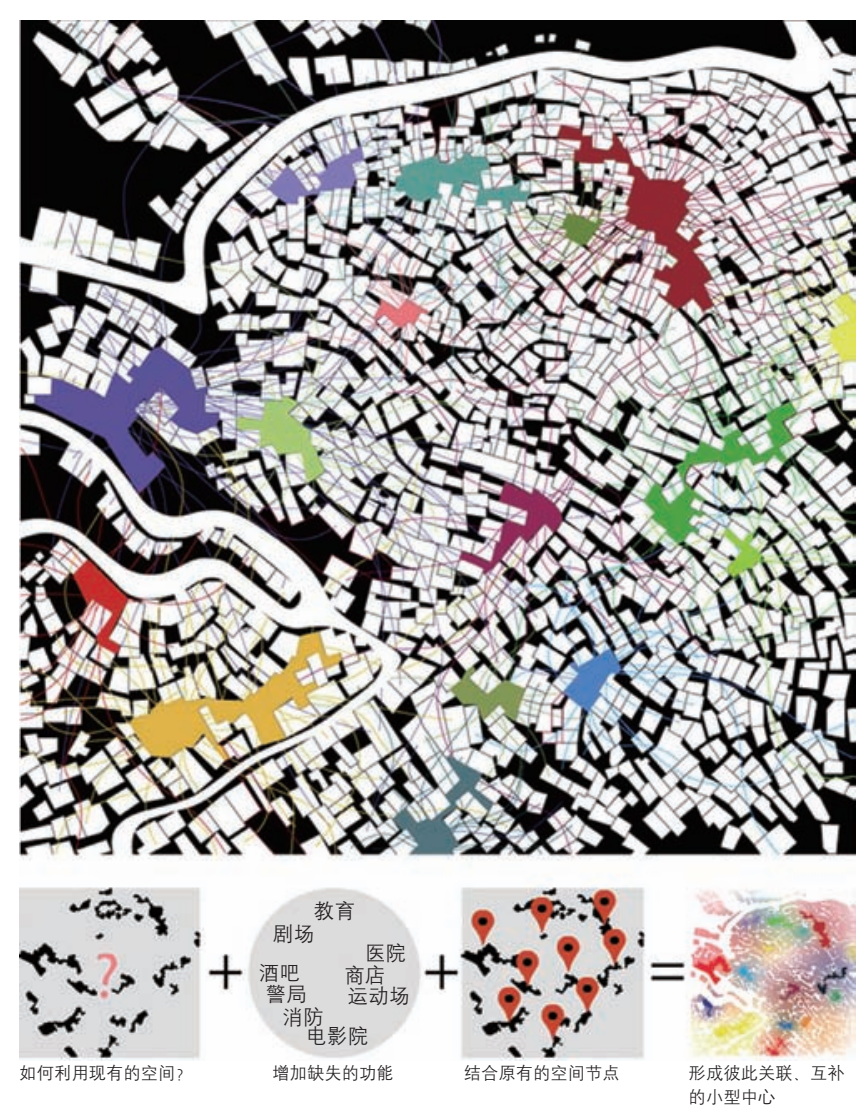

图 4 罗西尼亚不同的小型社区中心相互连接、功能互补

资料来源: 作者整理自 VINNITSKAYA I . Regeneration of the favela de rocinha slum / jan kudlicka[EB/OL]. (2011-07-01)[2017-05-26]. http://www.archdaily.com/ ; KUDLICKA J. Favela rocinha[EB/OL]. (2014-10-04)[2017-05-26]. https://www.area-arch.it/.

\section{3 新中产阶级的出现}

在发达的二次浪潮城市, 人们热衷于追逐属于自己的中 产阶级标签一郊区住宅、汽车, 这个巨大而昂贵的系统需 要高比例税收来支撑。新兴城市通过更为经济的基础设施介 人的方式, 例如哥伦比亚麦德林 (Medellin) 的高架公共交 通系统, 以及巴西库里蒂巴 (Curitiba) 对快速公交的创新 性利用, 逐渐规避了洛杉矶式的高速公路狂欢。在新兴城市 中, 智能手机与电脑已经成为新中产阶级的标志 ${ }^{[22]}$ 。

新兴城市中, 财政资源被更有效地分配到教育孩子、买 房子以及购买经济保险中。一些南美城市例如麦德林和圣保 罗, 城市居民生活处于较一些非洲与亚洲城市更为成熟的阶 段。据统计，在巴西，2003 年贫民窟的居民有 33\% 为中产 阶级, 而到了 2013 年, 中产阶级达到了 $65 \%^{[23]}$, 这是一个 很快速的增长。相当多的居民已经拥有了自己的住房, 并且 有足够的经济保障来支持孩子接受教育 ${ }^{[8]}$ 。

通信技术的发展为他们提供了更好的教育条件与工作机 会, 这些都是促进这一部分城市居民在短时间成为中产阶级 的原因。世界银行曾指出, 数字化通信技术在扩展支付服务、 强化社会安全网络、创造新的经济活动机会以及贫民窟环境 改造等方面发挥着重要作用, 帮助改善了贫民窟中的贫穷人 口以及弱势人群的生活。在里约热内卢与圣保罗的贫民窟中, 非政府组织 (NGO) 创办了 110 所计算机科学与公民教育 学校。学校每年培训了超过 25000 名年轻学生, 教授他们 通信技术与技巧, 为他们提供更好的工作、教育以及改善生 活的机会。同时, 通过数字化通信技术, 许多贫民窟居民接 受了人权教育。这样的教育帮助他们远离毒品, 重燃了对正 规学校的兴趣, 提高了对个人能力的认知 ${ }^{[14]}$ 。

\section{3 扁平城市}

3.1 数字化通信技术的发展带来了城市的去中心化趋向

无论在成熟城市还是在新兴城市中, 通信技术的发展正 在改变着人们的工作与生活方式, 也正在成为塑造城市的主 要力量 ${ }^{[7]}$ 。世界范围内, 互联网与智能手机的技术变革与持 有量在快速增长 ${ }^{2}$ 。相应地, 移动基础设施正在迅速升级, 以便于高性能的数据服务和新的应用程序能够更好地服务全 球数十亿用户 ${ }^{[24]}$ 。数字化通信技术正在渗透到城市的物质结 构中。这些技术明显地和无形地形成了新的使用与建构城市 空间的路径 ${ }^{[7]}$, 并将影响既有城市形态与结构。

在城市中, 人、技术、场所的关系将被重新定义。无论 收人多少, 灵巧的移动设备已经成为标配, 人们可以在城市

(1) 扬 - 库里利卡指出过度集中使现代城市容易受到伤害——如果其中一个停止运转, 那么城市将是危险的。而在贫民窟这里所有的部分彼此交流 与合作。这意味着, 如果一个独立单元出现问题, 它可以被它周边其他部分所替代。

(2) 根据 2015 年爱立信移动的报告, 2014 年全球移动用户总数约为 71 亿。 
中的大部分场所彼此连接。以人为本的技术产生了更为丰富 的用户体验, “面对面” 不再是维持全日制的工作关系的必 要。这样的变化将降低人们的出行量, 并对现有城市公共设 施提出新的要求 ${ }^{[8]}$ 。总有一天, 它会改变所有城市的面貌。

在数字化通信技术的影响下, 城市在近几十年呈现出更 为多中心和动态的形式趋势 ${ }^{[25]}$ 。相较于机动交通, 数字化 通信技术的发展带来了更具有革命性的城市空间的变化。新 的通信技术的发展可能引发工作场所位置的更为广泛的离散 ${ }^{[26]}$, 并会引发城市空间离散的趋势, 进一步地产生城市的“去 中心化浪潮” ${ }^{[7]}$ 。

克里斯多弗 - 亚历山大 (Christopher Alexander) 与阿 兰・柏图（Alain Bertaud）曾经梳理了不同类型城市模式的 特征, 并得到了相似的结论。亚历山大在对比了人造城市与 自然生长城市的组织方式的基础上, 解释了 “树形城市” 与 “半网格城市” 的不同概念 ${ }^{[27]}$ 。相似地, 柏图将最为常见的 城市空间结构归为四类, 其中最为典型的为 “单中心模式” 与“都市村庄型多中心模式” 1 $[9]$ 。虽然他们的出发点与表 述方式不尽相同，但是可以看出亚历山大的“半网格模式” 与柏图的“都市村庄型多中心模式”都表现出了“去中心 化”的特征, 它们都能够避免大量的城市资源集中于一个或 几个城市中心, 降低人们的通勤量, 是更为可持续的城市模

\section{表 1 亚历山大与柏图的城市模式对比}

\begin{tabular}{l|l}
\hline \multicolumn{1}{c|}{ 亚历山大的城市模式 } & \multicolumn{1}{c}{ 柏图的城市模式 } \\
\hline $\begin{array}{l}\text { 树形模式: 人为建造的城市, 分层级、节 } \\
\text { 点的单向关联 } \\
\text { 施高度集中的高密度中心, 人们 } \\
\text { 从城市周边向中心辐射状运动 }\end{array}$ \\
\hline$! ?$ 工
\end{tabular}

半网格模式：自然生长的城市, 弱层级、都市村庄型多中心模式：居住与 节点的多向关联 工作场所相邻, 人们步行或骑自 行车去工作（在现实中并不存在）

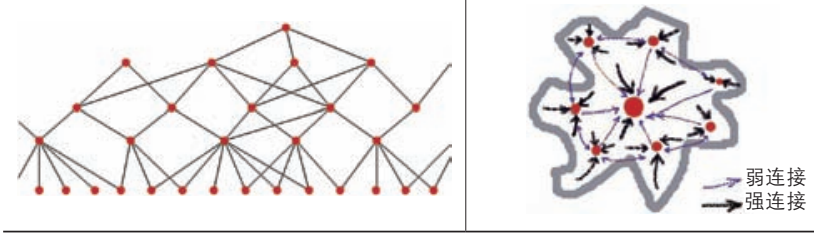

资料来源: 左侧图片来自ALEXANDER C. A city is not a tree [M] // LARICE M, MACDONALD E. The urban design reader. Abingdon: Routledge, 2007: 152-166 ; 右侧图片来自 BERTAUD A. The spatial organization of cities: deliberate outcome or unforeseen consequence? [R]. World Development Report 2003: Dynamic Development in a Sustainable World, World Bank, 2003.
式 (表 1)。

\section{2 扁平城市}

数字化通信技术进步对城市发展的影响指向了一个城市 发展的新趋向——可以被称作 “扁平城市”。扁平城市中的 “扁平”并不主要指城市形态上的低矮、平坦, 它表达的是 城市组织结构上的 “扁平”。

在此, 借用组织学中的 “扁平” 来帮助理解扁平城市的 概念, 例如扁平与公司的机构结构相关联时, 是指通过减少 组织层次、裁剪冗余人员来建立一种紧凑的扁平型组织结构, 使组织变得更为灵活、敏捷, 提高组织效率和效能 ${ }^{[28]}$ 。传统 的多层次、等级分明的金字塔企业组织结构因为笨重、迟缓 和缺乏灵活性而饱受批评, 许多发达国家企业已经向扁平化 的方向转变。

从城市空间的组织上来讲, 第二次浪潮城市具有明显的 金字塔状的层级关系，可以被称为 “极化城市”一城市的 主要工作、消费娱乐场所集中于 “极点”一一城市中心。人 们沿着城市道路向 “极点” 流动。拥堵的交通、大量耗散 的时间, 使城市陷人困境, 这样的局面在中国有愈演愈烈 的趋势。

与组织管理学中的 “扁平” 相类似, 扁平城市表达的是 一种层级更少、交互更多的城市组织方式。扁平城市是一种 在城市中大部分区域都能够获得经济与信息自由的城市。在 扁平城市中, 数字化通信技术为人们提供了摆脱或者至少是 极大地消减了对城市地理 “极点”依赖的条件。数字化通信 技术形成的虚拟连接部分代替了空间实体上的联系, 它能够 避免过多的城市资源集中于城市中心, 以及对昂贵的长距离 延伸基础设施的需求。这是一个去中心化的过程, 原来集中 于城市中心的公共空间与工作场所, 被分散到城市中的不同 区域, 人们的大部分工作、娱乐与居住等日常活动需求能够 在短距离、结构更为紧凑的城市空间 (多功能的街道与开 放空间）中解决。这样的趋势有利于产生一个更为可持续的 “扁平”的城市形态 (表 2)。在扁平城市中, 个体具有更 高的灵活性, 很多时候他并不需要驾车花大量的时间奔赴工 作所在的 CBD, 而只是步行去街角的小咖啡馆工作。这个 城市形态有助于降低城市的人均温室气体排放。形式上, 扁 平城市主要具有以下特征 :

（1）去中心化的城市结构 ;

（2）短距离的通勤模式;

（3）更小的城市公共中心。这些公共中心彼此连接、功 能互补, 能够容纳工作、娱乐等多项功能。

(1) 柏图将常见的城市空间结构分为单中心模式、都市村庄型多中心模式、随机运动型多中心模式以及类向心与随机运动型的单、多中心混合模式。 
在目前阶段, 一个完全意义上的现代扁平城市并不存在, 但是 “扁平” 的趋向是合理且不可避免的。

\section{4 扁平城市对中国城市发展的启示}

伴随着中国经济的快速发展以及城市化进程的深人, 中 国出现了住宅的郊区化、职住分离、通勤距离与时间增长以 及道路等基础设施的大量建设等现象。城市发展态势与发达 国家的第二次浪潮城市形式更为接近。

近些年来, 数字化通信技术的发展正在迅速地改变着中 国人的工作与生活。据有关数据, 截至 2016 年 6 月, 全国 互联网普及率达到 $51.7 \%$ 。移动电话 $4 \mathrm{G}$ 用户达到 7.14 亿年年。 虽然, 大部分单位远程办公日常化还是难以实现的。但是可 以看到, 微信与 $\mathrm{QQ}$ 等社交软件的广泛使用, 使一些单位的 部分日常工作从线下转到了线上。越来越多的人从事全职或 者兼职的线上工作，他们可以通过网络与其他工作伙伴以及 雇主联系; 淘宝、京东、美团等电商的兴起, 使网上购物、 订餐成为中国人 (尤其是 50 岁以下的能够熟练使用电脑与 智能手机的人群) 日常生活的常态。低廉的运输费用, 以及 较实体商场、餐厅更为优惠的价格、更为丰富的选择类型, 使网络购物量逐年上升。这样的局面也吸引了越来越多的独 立从业者借助网络从事商业经营。这是一项低门槛的商业活 动, 经营者无需具备大量的初始资金。它在商品类型与规模 等方面, 拥有更高的灵活度与自由度。

2015 年, 中国政府的《关于积极推进 “互联网 +” 行动 的指导意见》指明, 互联网的技术进步与社会各领域的深度 融合已经成为一项国家发展战略 ${ }^{[301}$ 。将互联网作为城市生产、
生活要素共享的重要平台, 为城市资源配置的优化与城市扁 平化发展提供了国家政策背景。

现阶段, 中国城市发展已经开始由大幅外扩转向对已有 城市建城区的修复与重构。在中国城市发展的现实背景下, 结合数字化通信技术的进步, 实现向扁平城市的转变, 以下 问题值得进一步研究。

\section{1 城市组织方式由 “职能中心” 向“综合组团”转变}

1990 年代以来, 为适应城市功能日趋复杂的发展要求, 中国大、中型城市多为多中心结构。城市中心功能趋于多样 化与专业化 ${ }^{[31]}$, 如城市商业中心、商务办公、文化中心、体 育中心、会展中心等。这些城市的空间结构基本上还是 “不 均衡式的多中心结构”, 居住区的空间分布是向城市边缘无 休止地扩张 ${ }^{[22]}$, 向心的交通压力依旧巨大。

从城市组织上看, 减小城市职能中心对城市日常生活 的主导程度, 梳理与补充不同城市建成区的城市功能与基 础设施, 形成更多规模适宜、拥有本地基础设施的综合组团, 这样的形式有利于建构人性化的适合步行的城市空间尺度, 弱化城市中区域间竞争的关系。数字化通信技术能够极大 提高城市不同区域的区位自由度和空间相互作用, 从而导 致工作职位对空间依赖程度逐步降低 ${ }^{[33]}$ 。疏散各城市职能 中心, 结合城市主要产业空间的分配, 形成多个紧凑的综 合工作、居住与休闲功能的综合城市组团。综合组团能够 有效减弱城市交通的向心运动, 降低城市的平均通勤时间 与拥堵几率。这是一个去中心化的过程, 也是中国城市向 扁平发展的一个重要趋势。

\section{表 2 组织结构与城市结构的对比}

分层的结构

资料来源 : 左侧图片来自于 MORGAN J. The 5 types of organizational structures: part 2, ‘flatter’ organizations [EB/OL]. (2015-07-08)[2017-03-26]. https://www.forbes.com/. ; 右侧图片为作者绘制 


\section{2 城市道路由运输型向生活型转变}

近些年中国城市道路的建设一直是围绕着 “机动车通 行” 这个中心进行的, 这是以牺牲街道上其他活动为代价的。 从道路横断面的划分到道路设施的配置、道路空间的塑造等, 体现出面向单一车行功能设计趋向, 以保证城市的高效运作 ${ }^{[34]}$ 。 这种以机动车运输为主导的道路呈现出尺度非人性化、空气 噪音污染严重、对行人具有危险性等问题, 将人行活动驱离 城市道路, 而这种活动恰恰是健康的扁平城市经济与社会文 化所需要的。

人们通勤距离的降低能够减少城市道路的建设量, 并使 更多的道路由运输型向生活型转变。生活型道路能够为人们 的 “工作、娱乐、居住于此处” 提供适宜的场所。中国城市 总体密度较高（这往往也意味着城市空间的高价值）、空间 狭促, 从经济、社会以及生态的角度, 利用城市道路构建或 者重建日常公共生活空间体系不失为一种好的选择。而如何 整体协调道路车行、人行空间, 沿街道布置商业服务设施、 公共绿地等城市公共设施, 为人们近距离解决工作、购物、 休闲等需求提供空间, 是值得进一步研究的课题。

\section{3 发展彼此关联、功能相补的小型城市中心}

集中了多样化、小规模服务设施的小型城市中心的发展, 对中国城市的扁平化非常重要。快速的城市化为中国城市带 来了大量的移民, 小型、独立的经济实体可以为他们提供更 多工作选择。小规模（lighter）、低成本（cheaper）的商业服 务设施集中于小型城市中心, 既可以为人们提供多样化的服 务与工作职位, 同时也意味着低风险、低成本。它占用较少 的时间、金钱, 可以快速与低代价地转换经营内容, 具有很 高的灵活性 ${ }^{[35,36]}$, 适合于资源有限的中国城市。近些年, 在 中国蓬勃发展的电子商务平台, 为这种小规模、价格低廉的 商业服务设施提供了更好的供货与销售条件。

这些小型中心彼此关联、补充，形成更为复杂与全面的 城市功能, 有利于在近距离为城市居民的日常工作与生活提 供更多、更为恰当的城市公共场所与设施, 进一步促进城市 的扁平化。

\section{5 结语}

扁平城市是一种数字化通信技术进步带来的城市发展趋 向, 它能够弱化城市中不同区域之间的竞争, 加强城市功能 的合理混合, 鼓励人们就近解决工作与娱乐的需求, 减少基 础设施建设量 (尤其是城市道路), 有效节约国家土地与能 源等重要资源, 是一种更为可持续与低碳的城市形式。扁平 城市对土地与能源相对紧张的中国城市今后的发展具有一定 的参考意义。UPI

\section{参考文献}

[1] United Nations. World urbanization prospects: the 2001 revision[M]. New York: United Nations, 2001

[2] WACHS M. Autos, transit, and the sprawl of Los Angeles: the 1920s[]]. Journal of the American Planning Association, 1984, 50(3): 297-310.

[3] DUTZIK T, BAXANDALL P. A new direction, our changing relationship with driving and the implications for America's future[J]. Black Enterprise, 2013, 47(4):141-142

[4] DAVIS B, DUTZIK T. Transportation and the new generation, why young peopleare drving less and what it means for transportation policy[M]. Boston: MA. U.S. PIRG Education Fund Frontier Group, 2012.

[5] SALOMOM I, MOKHTARIAN P L. Why don't you telecommute?[J]. Accee, 1997(10): 27-29.

[6] SALOMOM I, MOKHTARIAN P L. Can telecommunications help solve transportation problems? a decade later: are the prospects any better?[M] // DAVID A H, KENNETH J B. Handbook of transport modelling. Bingley: Emerald Group Publishing, 2007: 519 - 540.

[7] SHEN Q. Transportation, telecommunications, and the changing geography of opportunity[]]. Urban Geography, 1999, 20(4): 334-355.

[8] CHANDLER L, ROSS P. The anywhere working city [J/OL]. [2017-05-16]. http:// www.theanywhereorganisation.com.

[9] BERTAUD A. The spatial organization of cities: deliberate outcome or unforeseen consequence? [R]. World Development Report 2003: Dynamic Development in a Sustainable World, World Bank, 2003.

[10] MELOL M. Brazil: gains and losses in the Favelas[C] // NARAYAN D, PATESCH P, et al. Voices of the poor: from many lands. New York: Oxford University Press, 2002: 363-383.

[11] SAUNDERS D. Arrival city: the final migration and our next world[M]. Toronto: Vintage Canada, 2010.

[12] WYCHE S. Exploring mobile phone and social media use in a Nairobi slum: a case for alternative approaches to design in ICTD [C] // ICTD ' 15 Proceedings of the Seventh International Conference on Information and Communication Technologies and Development. 2015.

[13] NOVAIS A. Consumer behavior in the Favelas[OL]. The Brazil Business, 2012 [2017-05-22]. http://thebrazilbusiness.com/.

[14] RELHAN G, IONKOVA K, HUQUE R. Good urban governance through ICT: issues, analysis, and strategies[R]. Washington DC: World Bank, 2015: 24

[15] Improving the lives of slum dwellers through innovative uses of ICT[J/OL]. ITU News, 2010(8). [2016-04-23]. http://itunews.itu.int/.

[16] TOURE H. How mobile broadband can transform Africa[EB/OL]. CNN, (2012-02-27)[2017-05-22]. http://edition.cnn.com/.

[17] FOSTER C, HEEKS R. Researching ICT micro-enterprise in developing countries: themes, wider concepts and future directions[J]. The Electronic Journal on Information Systems in Developing Countries, 2010, 43(7): 1-20.

[18] ILAHIANE H, SHERRY J. Joutia: street vendor entrepreneurship and the informal economy of information and communication technologies in Morocco[J]. Journal of North African Studies, 2008, 13(2): 243-255.

[19] LUGO J, SAMPSON T. E-Informality in Venezuela: the 'other path' of technology[J]. Bulletin of Latin American Research, 2008, 27(1): 102-118.

[20] VOIGT K. Mobile phone: weapon against global poverty[EB/OL]. CNN, (2011-10-09)[2017-05-23]. http://www.cnn.com/.

[21] KUDLICKA J. Favela Rocinha[EB/OL]. (2014-10-04)[2017-05-26]. https:// www.area-arch.it/.

[22] ZIZOLA F. Brazil new middle class[EB/OL]. [2017-05-26]. http:// noorimages.com/.

[23] MEDEIROS I. Consumer behavior in Brazil: middle class in slums rises from $33 \%$ to $65 \%$ in 10 years[EB/OL]. (2014-03-04)[2017-05-26]. http:// www.designative.info/.

[24] The role of ICT in the proposed urban sustainable development goal and the new urban agenda[R]. UN HABITAT for a Better Urban Future. 2015. 
[25] ANAS A, ARNOTT R, SMALL K A. Urban spatial structure[J]. Journal of Economic Literature, 1998, 36(3): 1426-1464.

[26] HALL P. Revisiting the nonplace urban realm: have we come full circle?[J]. International Planning Studies, 1996, 1(1): 7-15.

[27] ALEXANDER C. A City is not a tree [M] // LARICE M, MACDONALD E. The Urban Design Reader. Abingdon: Routledge, 2007: 152-166.

[28] 张晓全, 曹光明. 扁平化一一西方企业组织结构的演变趋势 [J]. 管理现 代化，1994(6): 58-64.

[29] 中国网民规模达 7.1 亿互联网普及率 51.7\%[EB/OL]. (2016-08-03)[2017. 01-13]. http://www.chinanews.com/.

[30] 国务院。国务院关于积极推进 “互联网 +”行动的指导意见 $[E B / O L]$. (2015-07-04)[2017-01-04]. http://www.gov.cn/.
[31] 熊国平.90 年代以来我国城市形态演变的特征 [J]. 新建筑, 2006(3): 18-21.

[32] 韦亚平, 赵民. 都市区空间结构与绩效 [J]. 城市规划, 2006, 30(4): 9-16.

[33] 陈虹, 刘雨菡。“互联网+”时代的城市空间影响及规划变革 [J]. 规划 师, 2006, 32(4): 5-10.

[34] 马强.走向 “精明增长”一从一“汽车城市” 到“公共交通城市” $[\mathrm{M}]$. 北京: 中国建筑工业出版社, 2007: 190-191.

[35] CONDON P M. Seven rules for sustainable communities[M]. Washington DC: Island Press, 2010: 49.

[36] Project for Public Spaces. Placemaking and the future of cities[EB/OL]. (2012-11-13)[2017-01-20]. https://www.pps.org/.

(本文编辑：许玫)

\section{$>>$ 译者随笔 叶齐茂}

\section{鲁滨逊和《现代城市公共艺术》}

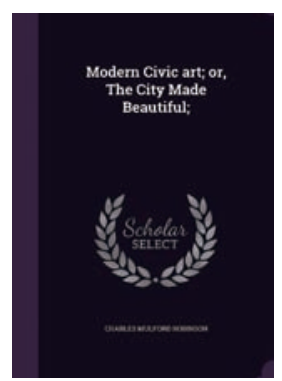

最近, 我开始翻译 20 世纪初城市美化思潮的教父, 鲁滨 逊（Charles Mulford Robinson）的《现代城市公共艺术》、《城 市规划》和《城市改造》三本书中的第一本, 于是, 久违了 的 “城市建设艺术” 或 “城市公共艺术”, 自然跃然纸上了。

鲁滨逊的兴趣在于审美, 但是, 他对城市的观察不同于 唯美主义者的观察。他这样写道, “城市不是造出来供观赏的, 城市造出来是为了生活的……直到我们把美丽的道路具体化 成为好的道路和清洁的道路, 否则, 优美道路的愿望总会留 在梦幻里。”他写道, 边远的居住区应该有 “宽阔的道路和狭 窄的道路, 笔直的道路和弯曲的道路, 有规律地散布着开放 空间的建成区, 那里有儿童游戏场所, 或供所有人使用的花 园。”他没有忽视美国城市的贫民窟, 他认为, 必须大规模改 造那些城市贫民窟, “直到审美精神降临到贫民窟, 那里也 有艺术的冲动在发挥作用, 否则, 美好的城市总是不完整的。”

鲁滨逊的这些著作反映了 20 世纪美国对城市规划的需 要, 也是第一次对广大民众谈起城市规划、城市美化或城市 公共艺术的问题。他写道, “除非我们认识到使用艺术的方 式去规划城市, 否则, 我们不会让城市真正美丽起来。”这 是对 19 世纪自由放任态度的意义深刻的批判, 19 世纪美国
城市建设者采用的就是这种自由放任的态度, 那时的城市建 设者遗忘了或忽视了前一辈人的成就。大型城市公园观念的 先驱奥姆斯特德甚至设想不优美的城市围绕着公园。鲁滨逊 认为, 那样的城市还不够美好。

鲁滨逊所描述和积极倡导的城市改造, 引起了全国范围 的兴趣, 这就产生了所谓的 “城市美化” 思潮。虽然鲁滨逊 在 “大西洋”系列文章中使用了 “城市美化” 这一术语, 但 是, 他是在 “公共艺术” 的意义上使用 “城市美化” 的, “公 共艺术” 的意义在于公益精神。因为当时正好发生了两个广 为宣传的国家事件 “芝加哥世界博览会” 和 “华盛顿中心区 规划”, 它们让审美问题凸显出来。城市美化抓住了公共艺 术的 “美” 的方面, 美丽的城市成了大众憧憬的事物。

《现代公共艺术》第一章的标题就是 “城市的新时代”。 于是, 我就想到, 我们现在不是正在建设新时代的城市吗? 这种相似难道是字面上的? 当然不是, 鲁滨逊的一些判断对 我们不无启发, 至少可以让我们把事情做得更好些, 帮助动 员更多的老百姓参与到美化城市的过程中来, 而不是较劲甚 至抵触。鲁滨逊是个报人, 他真的很懂如何动员民众, 而我 们本来是有群众路线这个法宝的, 可是, 一些人忘了使用它。 为什么不读读《现代城市公共艺术》?

《现代城市公共艺术》(1918 第四版) 出版整整 100 年了。 我们的规划教科书上总有关于城市美化思潮和鲁滨逊的内容, 但是, 真正读过原著的人胗胗无几。在翻译这本书的时候, 我 也在关注北京最近发生的一些事, 我每每想到, 芒福德的确说 的不错, 鲁滨逊的《现代城市公共艺术》“是他那个时代的杰作, 至今值得翻阅。”难道不是吗? UP 